\title{
Bioaccumulation des métaux lourds dans les tissus de deux espèces de Cichlide (Hemichromis fasciatus et Tilapia zillii $\times$ Tilapia guineensis) pêchés dans la partie ouest de la lagune Ebrié, Côte d'Ivoire.
}

\author{
KOUAMENAN N'zebo Moïse ${ }^{12}$, COULIBALY Safiatou2 * , ATSE Boua Celestin², GOORE Bi Gouli1 \\ ${ }^{1}$ Laboratoire d'Hydrobiologie et d'Eco-technologie des Eaux, I'UFR Biosciences, Université Félix Houphouët-Boigny \\ 2 Département Aquaculture, Centre de Recherches Océanologiques, BPV 18, Abidjan, Côte d'Ivoire \\ *Auteur correspondant : safinanga@gmail.com / 0022509974200
}

Original submitted in on $6^{\text {th }}$ February 2020. Published online at www.m.elewa.org/journals/ on $30^{\text {th }}$ April 2020 https://doi.org/10.35759/JABs.148.6

\begin{abstract}
RESUME
Objectif: Cette étude vise à évaluer l'influence des activités exercées en zones continentale et maritime de la partie ouest de la lagune Ebrié (Côte d'lvoire) sur la bioaccumulation de quatre métaux lourds $(\mathrm{Hg}, \mathrm{Pb}$, $\mathrm{Cd}$ et As) dans le muscle, le foie et le rein de deux (02) espèces de Cichlidae (Hemichromis fasciatus et Tilapia zillii × Tilapia guineensis).

Méthodologie et résultats: Les espèces de poissons ont été échantillonnées mensuellement de février 2014 à janvier 2015 sur cinq (05) stations. La sélection des stations est basée sur l'importance de la mortalité des poissons enregistrée en début de saison des pluies en 2013. Le dosage des métaux lourds dans les organes de poissons a été effectué selon les méthodes établies par EPA (2007). Les résultats montrent que l'espèce $H$. fasciatus accumule plus de métaux lourds dans ses organes que l'espèce $T$. zillii $\times T$. guineensis. Toutefois, le niveau de concentration des métaux lourds étudié est plus élevé dans les organes des deux espèces pendant la saison pluvieuse et la saison des crues. Par ailleurs, le niveau de concentration de l'arsenic dans les organes est plus élevé comparativement aux autres métaux lourds étudiés. Le test d'ANOVA a montré qu'il existe une différence significative entre la zone continentale et la zone maritime. Les espèces de poissons pêchés dans la zone maritime accumulent plus de métaux lourds dans leurs organes que celles collectées dans la zone continentale.

Conclusion et application des résultats: Cette étude a permis de comprendre que les organes des poissons pêchés dans les zones sont plus contaminés en métaux lourds pendant la saison pluvieuse et la saison des crues. Toutefois, les activités anthropiques dans les zones continentale et maritime induisent une contamination élevée des organes. Le niveau de concentration de cadmium et de l'arsenic dans le muscle des deux espèces de poisson dépasse la limite recommandée $(\mathrm{Cd}=0.05 \mathrm{mg} / \mathrm{kg}, \mathrm{As}=0.1 \mathrm{mg} /$ $\mathrm{kg})$ par WHO/FAO $(1995,2005)$. Cette forte concentration en $\mathrm{Cd}$ et As dans le muscle peut directement influencer la santé humaine par la consommation des poissons pêchés dans cette partie de la lagune Ebrié. Pour limiter la mortalité des poissons et garder la santé des consommateurs, il est important d'abandonner certaines pratiques culturales telles que l'utilisation abusive des produits phytosanitaires et de bateaux motorisés hors-bord dans cette partie de la lagune Ebrié.
\end{abstract}

Mots clés : Métaux lourds, organe, Cichlidae, zone continentale et maritime 
Bioaccumulation of some heavy metals in the tissues of two species of Cichlidae (Hemichromis fasciatus and hybrid Tilapia zillii $\times$ tilapia guineensis) collected from the western part of the Ebrie lagoon, Côte d'Ivoire

\begin{abstract}
Objectives: this study aims to evaluate the maritime and continental activities influence of the western part of Ebrie lagoon (Côte d'Ivoire) on the bioaccumulation of four heavy metals ( $\mathrm{Hg}, \mathrm{Pb}, \mathrm{Cd}$ and $\mathrm{As}$ ) in the muscles, liver and kidneys of two species of Cichlidae (Hemichromis fasciatus and the hybrid of Tilapia zillii $\times$ Tilapia guineensis)

Methodology and results: The fishes were sampled monthly from February 2014 to January 2015 at five (05) stations; site selection was based on the rate of deaths recorded in these areas in 2013. Heavy metals analyses of fish organs were carried out in accordance with EPA (2007) methods. The results showed that the species $H$. fasciatus accumulate more heavy metals in their tissues than the hybrid $T$. zillii $\times T$. guineensis. The highest concentrations in organs of both species are generally recorded during the rainy and swelling seasons. Arsenic is the most accumulated metal in the organs. ANOVA's analysis showed that there is a significant difference between the mainland area and the maritime area for all the heavy metals studied. Fish collected in the maritime area have the highest concentrations of heavy metals.

Conclusion of application of findings: this study showed that the fish organs contaminated in rainy and swelling seasons. Moreover, anthropic activities practiced in mainland and maritime areas induce organs contamination of heavy metals. The concentration level of $\mathrm{Cd}$ and $\mathrm{As}$ in the muscles of both species exceeds the recommended limit $(\mathrm{Cd}=0.05 \mathrm{mg} / \mathrm{kg}$, As $=0.1 \mathrm{mg} / \mathrm{kg})$ by WHO/FAO $(1995,2005)$. These high concentrations of heavy metals in the organs could affect the human health by fishes consumption collected western part of Ebrie lagoon. In order to limit the death of fishes and keep consumers healthy, it is important to abandon farm practices such as the abuse phytosanitary products and out board-motorized ship in this part of Ebrie lagoon.
\end{abstract}

Keywords: Heavy metals, organ, Cichlidae, continental and maritime area

\section{INTRODUCTION}

La lagune Ebrié, la plus importante du système lagunaire ivoirien, en raison de sa situation géographique et son importance économique est soumise à une forte pression anthropique (Arfi et Guiral, 1994). Les activités anthropiques sur les flancs continental et maritime dans la partie ouest de la lagune Ebrié contribuent à la dégradation de la qualité de l'eau. En effet, Cette partie de la lagune est bordée de grandes plantations de coco, de banane, de palmier, d'ananas et d'hévéas et enregistrent plusieurs villages de pécheurs. De plus, des activités intensives de pêche maritime sont menées par les populations riveraines et étrangères. Depuis 1999, des cas de mortalités de poissons ont été souvent signalées en début de saison des pluies. Au mois de mai 2013, ce phénomène s'est accentué avec une mort massive de poissons entrainant la fermeture de la pêche et toutes les fermes aquacoles (Koffi, 2013). De nombreuses études ont montré une pollution de l'eau et des sédiments de lagune Ebrié par les métaux lourds (Yao et al., 2007; Coulibaly et al., 2012; Koffi et al., 2014 ; Yapi, 2014 ; Daniel et al., 2016 ; Coulibaly et al., 2019). Les métaux lourds sont dangereux pour les espèces aquatiques à cause de leur persistance dans l'environnement, leur tendance à la bioaccumulation et surtout leur toxicité (Tabinda et al., 2010; Adama et al., 2015). Leur cible dans ces espèces est principalement le rein, le foie, les gonades, le muscle, les branchies et le cerveau. Par conséquent, les métaux lourds peuvent affecter non seulement la productivité et les capacités de reproduction mais aussi peuvent causer la mortalité des espèces (Claire et al., 2005; Koffi et al., 2014). Le niveau de bioaccumulation est élevé chez les espèces de poisson carnivores et se trouvant en bout de chaine alimentaire comme Hemichromis fasciatus 
(EFSA, 2005). Ainsi cette espèce de poisson apparait comme un bon indicateur de pollution métallique du milieu aquatique. $T$. guineensis $\times T$. zillii, des auteurs ont montré son abondance dans la partie ouest de la lagune Ebrié au cours ces dernières années (Boni et al., 2016). Compte tenu de cette abondance et de sa répartition dans la lagune Ebrié, cette espèce semble être un matériel biologique intéressant pour évaluer le niveau

\section{MATERIEL ET METHODES}

Echantillonnages et mesures des paramètres physico-chimiques: Les mesures des paramètres physico-chimiques, le prélèvement des eaux et des poissons ont été effectués mensuellement de février d'accumulation des métaux lourds. Le but de ce travail est d'évaluer l'influence des activités menées par les populations sur les flancs continental et maritime dans l'accumulation de mercure $(\mathrm{Hg})$, plomb $(\mathrm{Pb})$, cadmuim $(\mathrm{Cd})$ et l'arsenic (As) dans le muscle, le foie et le rein de deux espèces de cichlidae (Hemichromis fasciatus et Tilapia Guinensis $\times$ Tilapia Zillii) pêchées à l'ouest de la lagune Ebrié.

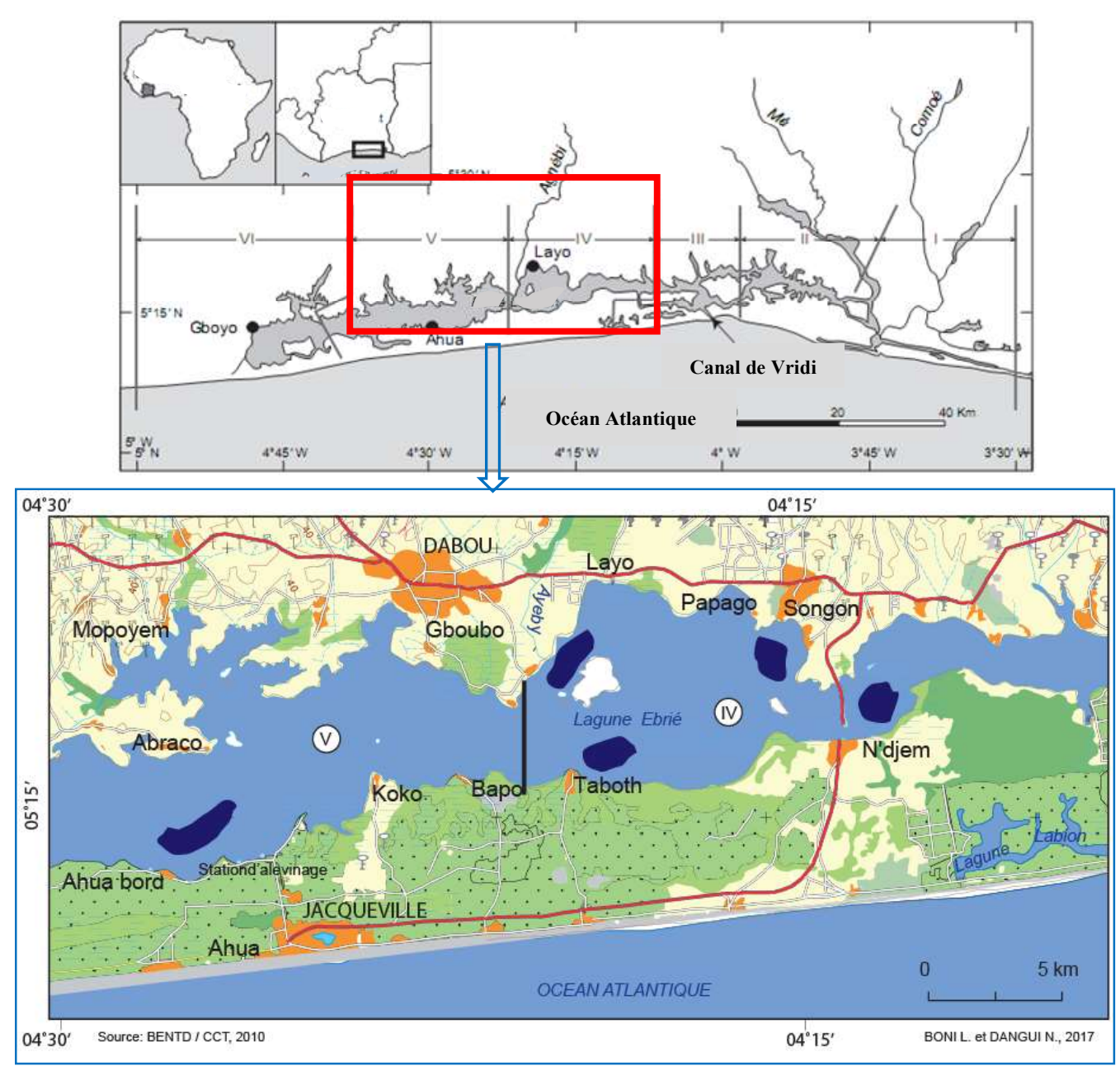

2014 à janvier 2015 sur cinq (05) stations (N'djem, Taboth, Ahua, Layo et Songon) des zones continentale et maritime à l'ouest de la lagune Ebrié $\left(5^{\circ} 13^{\prime} 15 \mathrm{~N}\right.$ and $4^{\circ} 42^{\prime} 0 "$ W) (Figure 1).

Figure 1: Localisation des stations d'échantillonnage dans les zones continentale et maritime à l'ouest de la lagune Ebrié (Côte d'Ivoire). (Songon, Layo,), maritime area (Ahua, Taboth, N'djem) 
Ces stations ont été sélectionnées en fonction de l'importance des activités agricoles et du degré de mortalité des poissons enregistré en mai 2013. Ainsi, Layo et Songon sont les stations de la zone continentale et N'djem, Taboth et Ahua sont retenues dans la zone maritime. L'eau est prélevée à l'aide d'une bouteille de NISKIN d'une capacité de 2,5 litres à une profondeur de $0,5 \mathrm{~m}$. Le prélèvement des poissons (Hemichromis fasciatus et Tilapia Guinensis × Tilapia Zillii) est effectué à l'aide des filets maillants, de la senne de plage et de l'épervier. Les filets maillants sont posés entre $16 \mathrm{~h}$ et $18 \mathrm{~h}$, relevés le lendemain entre $6 \mathrm{~h}$ et $8 \mathrm{~h}$ puis revisités entre $14 \mathrm{~h}$ et $16 \mathrm{~h}$ pour la pêche diurne. Au total 120 spécimens de chaque espèce de poissons sont capturés. Tous ces spécimens ont été transférés dans une glacière $\left(4^{\circ} \mathrm{C}\right)$ sous glace sèche jusqu'au laboratoire pour les analyses. Au cours des campagnes de prélèvements d'eau et de poissons, les paramètres physico-chimiques tels que le $\mathrm{pH}$, oxygène dissous, salinité, conductivité et la température ont été mesurés grâce à un multi-paramètre (YSI 6920). Ces mesures ont été effectuées in situ dans les $1 \mathrm{~m}$ de profondeur, entre $8 \mathrm{~h}$ et $10 \mathrm{~h}$. La transparence est également déterminée le disque de Secchi. Les concentrations des matières en suspension sont déterminées selon les prescriptions de Aminot et Chaussepied (1983).

\section{RESULTATS}

Paramètres de qualité: Les résultats de l'étude des paramètres de qualité de l'eau de la zone continentale montrent que la température oscille entre $27.91 \pm 1.45$ et $29.92 \pm 1.75^{\circ} \mathrm{C}$, le pH entre $6.80 \pm 0.59$ et $7.35 \pm 0.56$, l'oxygène dissous entre $5.29 \pm 1.79$ et $7.15 \pm 2.63 \mathrm{mg} / \mathrm{L}$, la salinité $1.05 \pm 1.18$ et $5.15 \pm 3.44 \%$, la conductivité entre $2071.19 \pm 2169.15$ et $9142.28 \pm 5749.33 \mathrm{mS} / \mathrm{cm}$, la transparence entre $0.77 \pm 0.32$ et $0.91 \pm 0.15 \mathrm{~m}$ et les matières en suspension entre $15.22 \pm 6.73$ et $16.89 \pm$ $9.90 \mathrm{mg} . \mathrm{L}^{-1}$ (Table 1). Dans la zone maritime, la variation saisonnière des paramètres de qualité de l'eau montre que la température varie de $28.38 \pm$ $1.35^{\circ} \mathrm{C}$ à $29.31 \pm 1,74^{\circ} \mathrm{C}$, le $\mathrm{pH}$ de $7.19 \pm 0.65$ à $7.32 \pm$ 0.28 , l'oxygène dissous de $5.39 \pm 2.21$ à $7.33 \pm 2.78$ $\mathrm{mg} / \mathrm{L}$, la salinité de $2.58 \pm 0.77$ à $4.56 \pm 3.34 \%$, la
Analyses chimiques des échantillons d'eau et d'organes de poissons: Les analyses chimiques de l'eau et des organes de poissons sont effectuées selon les méthodes établies par EPA (2007). L'eau prélevée sur les stations et filtrée est transportée au laboratoire pour la détermination des métaux lourds. Les concentrations des métaux lourds de l'eau tels que le $\mathrm{Pb}, \mathrm{Cd}$ et l'As sont déterminées au spectrométrie d'absorption atomique (Varian SAA) respectivement aux longueurs d'onde de 283,3 nm, 228,8 nm et $193,7 \mathrm{~nm}$. Le Hg de l'eau est déterminé à l'aide d'un spectrophotomètre d'absorption atomique (Shimadzu $A A 660)$ équipé d'un analyseur de vapeur froide de mercure à la longueur d'onde de 253,7 nm. Au laboratoire, les spécimens de poissons sont disséqués le foie et les reins sont extraits. En plus, cinq (05) gramme de muscle de poissons est prélevé. Après séchage et minéralisation des organes, la détermination des métaux lourds est effectuée selon la méthode EPA (2007).

Analyse des données : L'analyse de variance ANOVA a été utilisée pour tester les différences significatives entre les saisons, entre les zones continentale et maritime et entre les organes des espèces de poissons étudiés. Les différences sont considérées significatives à $p<0,005$.

conductivité de $5053 \pm 1249.23$ à $8272.21 \pm 5549.58$ $\mathrm{mS} / \mathrm{cm}$, la transparence de $1.27 \pm 0.40$ à $1.75 \pm 0.64 \mathrm{~m}$ et les matières en suspension de $11.50 \pm 6.06$ à $13.04 \pm$ $9.72 \mathrm{mg} / \mathrm{L}$ (Table 1). L'analyse statistique (ANOVA) montre une différence significative $(p<0.05)$ entre les saisons pour la salinité et la conductivité dans la zone continentale. Les valeurs élevées de ces paramètres sont observées pendant la saison sèche. Par ailleurs, la transparence et les matières en suspension sont statistiquement différente $(p<0.05)$ entre la zone continentale et la zone maritime. L'eau de la zone continentale est moins transparente avec des quantités de matières en suspension plus élevées que l'eau de la zone maritime. 
Kouamenan et al., J. Appl. Biosci. 2020 Bioaccumulation des métaux lourds dans les tissus de deux espèces de Cichlide (Hemichrom Tilapia guineensis) pêchés dans la lagune Ebrié, Côte d'Ivoire.

Table 1 : Valeurs moyennes annuelles et saisonnières des paramètres physico-chimiques mesurés dans l'eau de la zone continentale et de la zon de février 2014 à janvier 2015 à l'ouest de la lagune Ebrié

\begin{tabular}{lclll}
\hline & & & & Saisons (valeurs saisonnières : \\
\hline Paramètres & Zones & Annuelle & Sèche & Pluvieuse \\
\hline Température $\left({ }^{\circ} \mathrm{C}\right)$ & Zone continentale & $28.82 \pm 1.99$ & $29.92 \pm 1.75$ & $28.64 \pm 2.33$ \\
& Zone maritime & $28.90 \pm 1,67$ & $29.31 \pm 1,74$ & $28.99 \pm 1,87$ \\
\hline $\mathrm{pH}$ & Zone continentale & $7.07 \pm 0.56$ & $7.35 \pm 0.56$ & $6.80 \pm 0.59$ \\
& Zone maritime & $7.27 \pm 0.48$ & $7.30 \pm 0.46$ & $7.19 \pm 0.65$ \\
\hline Oxygène dissous $\mathrm{O}_{2}\left(\mathrm{mgL}^{-1}\right)$ & Zone continentale & $6.02 \pm 2.64$ & $7.15 \pm 2.63$ & $5.61 \pm 2.25$ \\
& Zone maritime & $6.15 \pm 2.66$ & $5.39 \pm 2.21$ & $7.33 \pm 2.78$ \\
\hline Salinité(\%o $)$ & Zone continentale & $2.88 \pm 1.18$ & $5.15 \pm 3.44 \mathrm{~b}$ & $2.63 \pm 3.68^{\mathrm{ab}}$ \\
& Zone maritime & $3.78 \pm 2,58$ & $4.56 \pm 3.34$ & $4.20 \pm 2.65$ \\
\hline Conductivité $\left(\mathrm{mS} . \mathrm{cm}^{-1}\right)$ & Zone continentale & $5474.64 \pm 5936.47$ & $9142.28 \pm 5749.33^{\mathrm{b}}$ & $5210.43 \pm 7024.44 \mathrm{ab}$ \\
& Zone maritime & $7175.10 \pm 4527.83$ & $8272.21 \pm 5549.58$ & $8200.08 \pm 5049.84$ \\
\hline Transparence $(\mathrm{m})$ & Zone continentale & $0.84 \pm 0.311$ & $0.91 \pm 0.15$ & $0.77 \pm 0.32$ \\
& Zone maritime & $1.44 \pm 0.48^{2}$ & $1.30 \pm 0.18$ & $1.27 \pm 0.40$ \\
\hline Matières en suspension $\left(\mathrm{mg} . \mathrm{L}^{-1}\right)$ & Zone continentale & $16.25 \pm 8.08^{2}$ & $16.89 \pm 9.90$ & $15.22 \pm 6.73$ \\
& Zone maritime & $12.40 \pm 7.691$ & $12.66 \pm 7.45$ & $13.04 \pm 9.72$
\end{tabular}

Les valeurs de la même ligne portant des lettres $a, b$ en exposant présentent une différence significative entre les saisons $(p<0,05)$ pour une zone donnée. Les val les chiffres 1 et 2 en exposant présentent une différence significative entre les zones $(p<0,05)$. 
Métaux lourds de l'eau : Les valeurs saisonnières des métaux lourds dans l'eau des zones continentale et maritime sont présentées dans le tableau 2. Ces valeurs varient dans la zone continentale de $2.15 \pm 1.17$ à $3.25 \pm 1.04 \mu \mathrm{g} / \mathrm{L}$ pour $\mathrm{Hg}$, de $2.38 \pm 1.13$ à $8.40 \pm 2.94$ $\mu \mathrm{g} / \mathrm{L}$ pour $\mathrm{Pb}$, de $2.63 \pm 2.06$ à $10.23 \pm 4.85 \mu \mathrm{g} / \mathrm{L}$ pour $\mathrm{Cd}$ et de $2.19 \pm 0.87$ à $3,53 \pm 1,18 \mu \mathrm{g} / \mathrm{L}$ pour As. Dans la zone continentale, les concentrations des métaux lourds de l'eau sont comprises entre $1.98 \pm 0.77$ et $2.09 \pm 0.65 \mu \mathrm{g} / \mathrm{L}$ pour $\mathrm{Hg}$, entre $3.65 \pm 1.53$ et $4.89 \pm 2,20$ $\mu \mathrm{g} / \mathrm{L}$ pour $\mathrm{Pb}$, entre $5.63 \pm 5.52$ et $11.48 \pm 10.48 \mu \mathrm{g} / \mathrm{L}$ pour $\mathrm{Cd}$ et entre $3.04 \pm 1.92$ et $5.28 \pm 2.85$ pour As (tableau 2). L'analyse statistique (ANOVA) montre une différence significative $(p<0.05)$ entre les saisons pour le plomb et le cadmium seulement en zone continentale. Les concentrations du plomb et du cadmium sont élevées pendant les saisons sèche et pluvieuse. Par ailleurs, les concentrations moyennes $d u$ plomb et de l'arsenic sont significativement différentes entre les deux zones. La zone continentale enregistre la valeur élevée en plomb. En revanche, la zone maritime enregistre la concentration élevée de l'arsenic (tableau 2).

Table 2: Valeurs moyennes annuelles et saisonnières des métaux lourds ( $\mu$ g.L-1) mesurées dans l'eau des zones continentale et maritime entre février 2014 et janvier 2015 à l'ouest de la lagune Ebrié

\begin{tabular}{llllll}
\hline & & \multicolumn{3}{c}{ Saisons (valeurs saisonnières \pm écart type) } \\
\hline Métaux lourds & Zones & Annuelle & Sèche & Pluvieuse & Crues \\
\hline Mercure $(\mathrm{Hg})$ & Zone continentale & $2.80 \pm 1.08$ & $3.01 \pm 0.8$ & $2.15 \pm 1.17$ & $3.25 \pm 1.04$ \\
& Zone maritime & $2.07 \pm 0.75$ & $1.98 \pm 0.77$ & $2.15 \pm 0.87$ & $2.09 \pm 0.65$ \\
\hline Plomb $(\mathrm{Pb})$ & Zone continentale & $6.36 \pm 3,832$ & $8.31 \pm 3.34 \mathrm{~b}$ & $8.40 \pm 2.94 \mathrm{~b}$ & $2.38 \pm 1.13 \mathrm{a}$ \\
& Zone maritime & $4.10 \pm 2.431$ & $3.65 \pm 1.53$ & $4.89 \pm 2,20$ & $3.76 \pm 3.26$ \\
\hline Cadmium (Cd) & Zone continentale & $7.57 \pm 6,13$ & $10.23 \pm 4.85 \mathrm{~b}$ & $9.85 \pm 7.35 \mathrm{~b}$ & $2.63 \pm 2.06 \mathrm{a}$ \\
& Zone maritime & $7.98 \pm 7.83$ & $11.48 \pm 10.48$ & $5.63 \pm 5.52$ & $6.85 \pm 5.86$ \\
\hline Arsenic $(\mathrm{As})$ & Zone continentale & $2.83 \pm 1.211$ & $2,79 \pm 1,30$ & $3,53 \pm 1,18$ & $2.19 \pm 0.87$ \\
& Zone maritime & $4.21 \pm 2.682$ & $4.32 \pm 2.88$ & $3.04 \pm 1.92$ & $5.28 \pm 2.85$ \\
\hline
\end{tabular}

Les valeurs de la même ligne portant des lettres $a$, $b$ en exposant présentent une différence significative entre les saisons $(p<0,05)$ pour une zone donnée. Les valeurs de la même colonne portant les chiffres 1 et 2 en exposant présentent une différence significative entre les zones ( $p$ $<0,05)$.

Métaux lourds dans les organes: L'analyse de variance (ANOVA) montre une variation saisonnière significative $(p<0.05)$ des métaux lourds dans les organes de Hemichromis fasciatus de la zone continentale excepté le $\mathrm{Hg}$ dans le muscle et le foie, ainsi que le $\mathrm{Pb}$ dans les reins. Les concentrations $\mathrm{du}$ $\mathrm{Pb}$ et de l'As sont élevées en saison des pluies dans les organes. Par contre, la concentration de $\mathrm{Cd}$ dans les organes est élevée en saison des crues. Le test de variance de l'ANOVA $(p<0.05)$ montre une différence significative entre les saisons pour le $\mathrm{Hg}$ et l'As dans le foie, le $\mathrm{Pb}$ et le $\mathrm{Cd}$ dans le muscle et le rein de Hemichromis fasciatus issu de la zone maritime. Le $\mathrm{Hg}$, le $\mathrm{Pb}$ et l'As sont élevés dans les organes pendant la saison pluvieuse. En revanche, le $\mathrm{Cd}$ enregistre sa valeur élevée dans les organes en saison sèche (tableau 3). L'analyse statistique (ANOVA) montre une différence significative $(p<0.05)$ entre les saisons pour le $\mathrm{Hg}$ et l'As dans le foie et l'As dans le rein de Tilapia Guinensis $\times$ Tilapia Zillii de la zone continentale. Les concentrations de $\mathrm{Hg}$ et l'As sont élevées dans les organes pendant les saisons sèches et des crues. Les variations saisonnières sont significativement différentes $(p<0.05)$ pour le $\mathrm{Hg}$ dans le rein et l'As dans le muscle de Tilapia Guinensis $\times$ Tilapia Zillii de la zone maritime. Les valeurs de $\mathrm{Hg}$ et l'As dans ces organes sont maximales en saisons sèche et des crues (tableau 3). Le test de l'analyse de variance montre une différence significative $(p<0.05)$ entre les organes pour tous les métaux lourds. Les valeurs moyennes des métaux lourds sont élevées dans le rein et basses dans le muscle. L'ordre d'accumulation des métaux dans les organes est Rein>Foie>Muscle. Les valeurs moyennes de tous les métaux lourds sont élevées dans l'ensemble des organes de Hemichromis fasciatus en comparaison avec celles observées dans les organes de Tilapia zillii $\times$ Tilapia guineensis. Une différence significative est notée entre la zone continentale et la zone maritime pour les métaux lourds dosés dans les organes des espèces de poissons. Cette différence d'accumulation des métaux lourds entre les zones est récurrente dans le foie et le rein des deux espèces de poissons étudiées. Par ailleurs, l'ordre de concentration des métaux lourds dans les organes de $H$. fasciatus et $T$. zillii $\times T$. guineensis s'établit de façon croissante: $\mathrm{As}>\mathrm{Pb}>\mathrm{Cd}>\mathrm{Hg}$ (tableau 4). 
Table 3 : Valeurs saisonnières des métaux lourds $\left(\mathrm{mg}^{\mathrm{kg}}{ }^{-1}\right)$ dans le muscle, le foie et le rein de Hemichromis fasciatus et Tilapia zillii $\times$ Tilapia guineensis pêchés entre janvier 2014 et février 2015 dans la zone continentale et la zone maritime à l'ouest de la lagune Ebrié

\begin{tabular}{|c|c|c|c|c|c|c|c|c|c|c|c|}
\hline \multirow[t]{2}{*}{ Espèces } & \multicolumn{5}{|c|}{ Muscle } & \multicolumn{3}{|l|}{ Foie } & \multicolumn{3}{|l|}{ Rein } \\
\hline & Zones & & SS & SP & SC & SS & SP & SC & SS & SP & SC \\
\hline \multirow{8}{*}{$\begin{array}{l}\text { Hemichromis } \\
\text { fasciatus }\end{array}$} & \multirow{4}{*}{ Continentale } & $\mathrm{Hg}$ & $0.17 \pm 0.02$ & $0.18 \pm 0.02$ & $0.18 \pm 0.01$ & $0.29 \pm 0.01$ & $0.27 \pm 0.05$ & $0.30 \pm 0.03$ & $0.31 \pm 0.05^{a}$ & $0.46 \pm 0.05^{c}$ & $0.37 \pm 0.02^{b}$ \\
\hline & & $\mathrm{Pb}$ & $0.14 \pm 0.02^{a}$ & $0.18 \pm 0.03^{b}$ & $0.18 \pm 0.01^{b}$ & $0.28 \pm 0.05^{\mathrm{ab}}$ & $0.26 \pm 0.05^{a}$ & $0.30 \pm 0.05^{b}$ & $0.49 \pm 0.09$ & $0.58 \pm 0.48$ & $0.48 \pm 0.17$ \\
\hline & & $\mathrm{Cd}$ & $0.11 \pm 0.05^{b}$ & $0.05 \pm 0.02^{a}$ & $0.08 \pm 0.05^{a b}$ & $0.16 \pm 0.05^{a}$ & $0.16 \pm 0.02^{a}$ & $0.28 \pm 0.05^{b}$ & $0.21 \pm 0.06^{a}$ & $0.24 \pm 0.04^{a}$ & $0.32 \pm 0.07^{b}$ \\
\hline & & As & $0.23 \pm 0.04 \mathrm{e}^{\mathrm{a}}$ & $0.38 \pm 0.14^{b}$ & $0.22 \pm 0.020^{a}$ & $0.37 \pm 0.052^{a}$ & $0.78 \pm 0.26^{b}$ & $0.70 \pm 0.15^{b}$ & $0.70 \pm 0.21^{a}$ & $2.04 \pm 0.35^{b}$ & $1.74 \pm 0.30^{b}$ \\
\hline & \multirow{4}{*}{ Maritime } & $\mathrm{Hg}$ & $0.19 \pm 0.03$ & $0.17 \pm 0.02$ & $0.16 \pm 0.04$ & $0.29 \pm 0.03^{b}$ & $0.36 \pm 0.05^{c}$ & $0.23 \pm 0.04^{a}$ & $0.43 \pm 0.05$ & $0.43 \pm 0.11$ & $0.36 \pm 0.07$ \\
\hline & & $\mathrm{Pb}$ & $0.18 \pm 0.02^{\mathrm{ab}}$ & $0,23 \pm 0.09 b$ & $0.16 \pm 0.03^{a}$ & $0.29 \pm 0.04$ & $0.35 \pm 0.17$ & $0.27 \pm 0.07$ & $0.39 \pm 0.07$ & $0.37 \pm 0.14$ & $0.39 \pm 0.09$ \\
\hline & & $\mathrm{Cd}$ & $0.40 \pm 0.50^{c}$ & $0.06 \pm 0.05^{a}$ & $0.12 \pm 0.03^{b}$ & $0.51 \pm 0.42$ & $0.29 \pm 0.08$ & $0.26 \pm 0.10$ & $0.80 \pm 0.50^{b}$ & $0.35 \pm 0.17^{a}$ & $0.33 \pm 0.09^{a}$ \\
\hline & & As & $0.23 \pm 0.03$ & $0.26 \pm 0.09$ & $0.31 \pm 0.19$ & $0.49 \pm 0.23^{a b}$ & $0.89 \pm 0.85^{b}$ & $0.26 \pm 0.13^{a}$ & $1.30 \pm 0.62$ & $1.21 \pm 0.93$ & $0.73 \pm 0.61$ \\
\hline \multirow{8}{*}{$\begin{array}{l}\text { Tilapia zillii × } \\
\text { Tilapia } \\
\text { guineensis }\end{array}$} & \multirow{4}{*}{ Continentale } & $\mathrm{Hg}$ & $0.11 \pm 0.04$ & $0.12 \pm 0.05$ & $0.11 \pm 0.03$ & $0.27 \pm 0.01^{b}$ & $0.18 \pm 0.06^{a}$ & $0.26 \pm 0.07^{b}$ & $0.27 \pm 0.02$ & $0.28 \pm 0.09$ & $0.30 \pm 0.07$ \\
\hline & & $\mathrm{Pb}$ & $0.09 \pm 0.05$ & $0.12 \pm 0.06$ & $0.12 \pm 0.02$ & $0.18 \pm 0.07$ & $0.14 \pm 0.10$ & $0.24 \pm 0,11$ & $0.37 \pm 0.06$ & $0.44 \pm 0.52$ & $0.39 \pm 0.16$ \\
\hline & & $\mathrm{Cd}$ & $0.06 \pm 0.05$ & $0.03 \pm 0.03$ & $0.02 \pm 0.01$ & $0.11 \pm 0.02$ & $0.10 \pm 0.07$ & $0.15 \pm 0.03$ & $0.12 \pm 0.06$ & $0.17 \pm 0.10$ & $0.21 \pm 0.06$ \\
\hline & & As & $0.16 \pm 0.03$ & $0.24 \pm 0.12$ & $0.17 \pm 0.05$ & $0.32 \pm 0.06^{b}$ & $0.21 \pm 0.12^{a}$ & $0.36 \pm 0.05^{b}$ & $0.33 \pm 0.16^{\mathrm{a}}$ & $1.35 \pm 0.72^{b}$ & $1.11 \pm 0.47^{b}$ \\
\hline & \multirow{4}{*}{ Maritime } & $\mathrm{Hg}$ & $0.15 \pm 0.04$ & $0.11 \pm 0.05$ & $0.12 \pm 0.05$ & $0.22 \pm 0.03$ & $0.22 \pm 0.09$ & $0.19 \pm 0.05$ & $0.34 \pm 0.05^{b}$ & $0.23 \pm 0.10^{a}$ & $0.36 \pm 0.10^{b}$ \\
\hline & & $\mathrm{Pb}$ & $0.13 \pm 0.03$ & $0.17 \pm 0.10$ & $0.15 \pm 0.07$ & $0.23 \pm 0.04$ & $0.22 \pm 0.10$ & $0.29 \pm 0.12$ & $0.29 \pm 0.12$ & $0.47 \pm 0.36$ & $0.32 \pm 0.11$ \\
\hline & & $\mathrm{Cd}$ & $0.05 \pm 0.03$ & $0.10 \pm 0.12$ & $0.08 \pm 0.07$ & $0.17 \pm 0.06$ & $0,18 \pm 0.10$ & $0.23 \pm 0.12$ & $0.30 \pm 0.09$ & $0.28 \pm 0.15$ & $0.32 \pm 0.15$ \\
\hline & & As & $0.16 \pm 0.04^{a}$ & $0.25 \pm 0.10^{b}$ & $0.12 \pm 0.04^{a}$ & $0.28 \pm 0.17$ & $0.64 \pm 0.60$ & $0.32 \pm 0.11$ & $0.85 \pm 0.50$ & $0.63 \pm 0.53$ & $1.21 \pm 1.05$ \\
\hline
\end{tabular}

Les valeurs de la même ligne portant des lettres $a, b$ en exposant présentent une différence significative entre les saisons $(p<0,05)$ pour une zone donnée. $(S S$ Saison Sèche ; SP Saison Pluvieuse ; SC Saison des pluies). 
Kouamenan et al., J. Appl. Biosci. 2020 Bioaccumulation des métaux lourds dans les tissus de deux espèces de Cichlide (Hemichromis fasciatus et Tilapia zillii × Tilapia guineensis) pêchés dans la lagune Ebrié, Côte d'Ivoire.

Table 4 : Valeurs annuelles des métaux lourds $\left(\mathrm{mg} \mathrm{kg}^{-1}\right)$ mesurées dans les organes de Hemichromis fasciatus et de Tilapia zillii × Tilapia guineensis pêchés entre janvier 2014 et février 2015dans la zone continentale et en zone maritime à l'ouest de la lagune Ebrié

\begin{tabular}{|c|c|c|c|c|c|}
\hline Métaux lourds & Espèces & Zones & Muscle & Foie & Rein \\
\hline \multirow{4}{*}{$\mathrm{Hg}$} & H.f & Zone continentale & $0,18 \pm 0,02^{a}$ & $0,29 \pm 0,04^{b}$ & $0,38 \pm 0,08^{1, c}$ \\
\hline & & Zone maritime & $0,18 \pm 0,03^{a}$ & $0,29 \pm 0,06^{b}$ & $0,41 \pm 0,092, \mathrm{c}$ \\
\hline & $\mathrm{Tz} \times \mathrm{Tg}$ & Zone continentale & $0,11 \pm 0,04^{a}$ & $0,24 \pm 0,07^{2, b}$ & $0,28 \pm 0,06^{1, c}$ \\
\hline & & Zone maritime & $0,12 \pm 0,05^{a}$ & $0,21 \pm 0.06^{1, b}$ & $0,31 \pm 0,10^{2, c}$ \\
\hline \multirow{4}{*}{$\mathrm{Pb}$} & H.f & Zone continentale & $0,17 \pm 0,03^{a}$ & $0,27 \pm 0,06^{1, a}$ & $0,52 \pm 0,292, b$ \\
\hline & & Zone maritime & $0,19 \pm 0,06^{a}$ & $0,30 \pm 0,11^{2, b}$ & $0,39 \pm 0,10^{1, b}$ \\
\hline & $\mathrm{Tz} \times \mathrm{Tg}$ & Zone continentale & $0,11 \pm 0,05^{1, a}$ & $0,19 \pm 0,10^{1, b}$ & $0,40 \pm 0,30^{2, c}$ \\
\hline & & Zone maritime & $0,15 \pm 0,072, a$ & $0,25 \pm 0,09^{2, b}$ & $0,36 \pm 0,241, \mathrm{c}$ \\
\hline \multirow{4}{*}{$\mathrm{Cd}$} & H.f & Zone continentale & $0,08 \pm 0,05^{1, a}$ & $0,20 \pm 0,07^{1, b}$ & $0,26 \pm 0,08^{1, c}$ \\
\hline & & Zone maritime & $0,19 \pm 0,32^{2, a}$ & $0,35 \pm 0,272, a b$ & $0,49 \pm 0,372, b$ \\
\hline & $\mathrm{Tz} \times \mathrm{Tg}$ & Zone continentale & $0,04 \pm 0,04^{1, a}$ & $0,12 \pm 0,05^{1, b}$ & $0,17 \pm 0,08^{1, c}$ \\
\hline & & Zone maritime & $0,08 \pm 0,082, a$ & $0,19 \pm 0.10^{2, b}$ & $0,30 \pm 0,13^{2, c}$ \\
\hline \multirow{4}{*}{ As } & H.f & Zone continentale & $0,28 \pm 0,11^{\mathrm{a}}$ & $0,62 \pm 0,25^{b}$ & $1,49 \pm 0,65^{2, c}$ \\
\hline & & Zone maritime & $0,27 \pm 0,12^{a}$ & $0,60 \pm 0,58 \mathrm{a}$ & $1,07 \pm 0,76^{1, b}$ \\
\hline & $\mathrm{Tz} \times \mathrm{Tg}$ & Zone continentale & $0,19 \pm 0,08^{a}$ & $0,29 \pm 0,10^{1, a}$ & $0,93 \pm 0,66^{2, b}$ \\
\hline & & Zone maritime & $0,18 \pm 0,09 a$ & $0,41 \pm 0,392, a$ & $0,90 \pm 0,76^{1, b}$ \\
\hline
\end{tabular}

Les valeurs de la même ligne portant des lettres $a, b$ en exposant présentent une différence significative entre les organes $(p<0,05)$ pour une zone donnée. Les valeurs de la même colonne portant les chiffres 1 et 2 en exposant présentent une différence significative entre les zones $(p$ $<0,05)$. H.f : Hemichromis fasciatus ; Tz x Tg : Tilapia zillii $\times$ Tilapia guineensis

\section{DISCUSSION}

Les résultats de cette étude ont montré que la température des eaux des zones d'étude est élevée durant la période d'échantillonnage. Cette chaude température des eaux semble dépendre du degré d'insolation et de la température élevée de l'air (Kouassi et al., 2005). Le caractère basique des eaux des zones continentale et maritime à l'ouest de la lagune Ebrié résulterait de la présence dans le sous-sol d'une formation de composé azoté organique (Arfi et Guiral, 1994). La salinité et la conductivité élevées observées dans les deux zones durant la saison sèche peuvent être due à l'évaporation maximale de l'eau durant cette période (Koffi et al. 2014). Cette étude a montré une différence significative entre les deux zones pour la transparence et les matières en suspension. Cette forte charge de matières en suspension de l'eau de la zone continentale serait liée aux matières issues des activités agricoles et charriées par les eaux intérieures. Selon Coulibaly et al. (2019), la zone continentale est influencée par des eaux météorites. En effet, selon une étude menée par Affian et al. (2008), ces eaux sont le principal pourvoyeur des matières en suspension de la lagune Ebrié. Les résultats de cette étude révèlent la présence des éléments traces métalliques dans les eaux des zones continentale et maritime. La variation saisonnière a montré une différence significative entre les saisons pour l'accumulation de plomb $(\mathrm{Pb})$ et de cadmium $(\mathrm{Cd})$ dans l'eau de la zone continentale. Les fortes valeurs sont enregistrées pendant les saisons sèche et pluvieuse. Cette différence saisonnière peut être attribuée au changement des propriétés physiques et chimiques. En effet, les taux élevés des métaux lourds des eaux de la zone continentale en saison sèche sont probablement dus à la réduction du volume d'eau (Obasohan et al., 2006). Par ailleurs, les fortes valeurs des métaux lourds pendant la saison des pluies seraient imputables aux déversements des eaux de ruissellement. Les eaux usées et les eaux de ruissellement chargées de déchets domestiques issus des détergents, des bactéries, des produits cosmétiques et d'autres produits hygiéniques utilisés par les populations environnantes sont les sources potentielles de la contamination de la lagune Ebrié par les métaux toxiques (Inza et al. 2015). Les résultats de cette étude montrent également une différence significative entre la zone continentale et la zone maritime pour le plomb et l'arsenic. Les concentrations élevées du plomb en zone continentale sont le résultat de l'accumulation de la pollution agricole et domestique. En effet, ces concentrations élevées proviendraient de l'utilisation de fongicides dans les plantations industrielles de cocotier (Adjagodo et al., 2016), et de rejet d'eau douce qui pourrait transporter une quantité importante de produits phytosanitaires 
issus des pratiques agricoles sur le bassin versant (Diomandé et al., 2009). Cependant, les teneurs moyennes des métaux lourds de cette étude sont basses comparativement aux concentrations $(\mathrm{Hg}=10$ $300 \mu \mathrm{g} / \mathrm{L} ; \mathrm{Cd}=20-260 \mu \mathrm{g} / \mathrm{L}$ et $\mathrm{Pb}=240-480 \mu \mathrm{g} / \mathrm{L})$ des métaux lourds trouvées par Coulibaly et al. (2012) dans la lagune Ebrié et aux concentrations $(\mathrm{Cd}=50 \mu \mathrm{g} / \mathrm{L}$ et $\mathrm{Pb}=70 \mu \mathrm{g} / \mathrm{L}$ et $\mathrm{As}=251 \mu \mathrm{g} / \mathrm{L})$ des métaux lourds trouvées par Sama et al. (2014) dans les eaux de la lagune d'Aného au Togo. Cette différence de concentrations de métaux serait liée à la situation géographique des lagunes et à l'impact des activités urbaines. L'accumulation des métaux lourds dans les organes de poissons est significativement différente d'une saison à une autre. Cette variation saisonnière de la concentration des métaux dans les organes résulterait de la modification de la température de l'eau et des facteurs intrinsèques aux poissons tels que les cycles de croissance et de reproduction (Dural et al., 2010). De plus, cette différence de concentrations saisonnières des métaux lourds dans les organes peut être due à une pollution locale. Le test statistique ANOVA a montré que l'accumulation des métaux lourds est significativement différente entre les organes. Le rein et le foie sont les organes les plus accumulateurs d'éléments métalliques tandis que le muscle est l'organe le moins accumulateur. Cette différence s'expliquerait par le fait que le rein et le foie sont métaboliquement actifs comparativement au muscle qui semble avoir un faible métabolisme (Filazi et al., 2003 ; Ploetz et al., 2007). Par ailleurs, l'affinité de ces organes avec les métaux lourds pourrait résider de leur capacité à induire une liaison métal-protéine comme dans le cas des métallothioneines (Canli et Atli, 2003). L'ordre de concentration des métaux lourds dans les organes montre que l'arsenic est le contaminant qui se concentre plus dans les organes. Cette forte contamination de l'arsenic comparée aux autres métaux lourds étudiés, peut-être dû à la disponibilité naturelle de ce métal dans notre zone d'étude. En effet, le sous-sol de la zone d'étude renferme de riches gisements de minéraux et d'hydrocarbures (FOXTROT, 2012). Islam (2016) rapporte que les taux élevés de l'arsenic dans les organes pourraient être dus à une plus grande capacité d'enrichissement de ce métal du milieu aquatique. De plus, l'absorption facile de l'arsenic par les espèces de tilapia faciliterait l'accumulation de ce métal dans leurs organes $(\mathrm{Wu}$, 2007). Dans cette étude les organes des poissons pêchés dans l'eau de la zone maritime ont des concentrations élevées en métaux lourds comparativement à ceux de la zone continentale. Les concentrations élevées de métaux lourds dans les organes des poissons pêchés dans la zone maritime sont probablement dues aux rejets constants de moteur et de mazout en raison de la forte circulation de bateaux motorisés hors-bord utilisés pour pêcher et transporter des personnes. En effet, Traore et al. (2016) et Gouin et al. (2016) rapportent que les huiles de moteur et de carburant sont les principales sources des métaux lourds dans la lagune, singulièrement dans les zones à forte circulation des bateaux motorisés. En outre, Calamari et Naeve, 1994 ont noté que les carburants contiennent de nombreux métaux lourds. L'analyse des résultats de cette étude a montré que les organes de Hemichromis fasciatus sont plus contaminés en métaux lourds. La contamination des organes de cette espèce de poisson par les métaux lourds serait liée à son régime alimentaire. Selon Blahoua et al. (2017), le spectre alimentaire de Hemichromis fasciatus est constitué de poissons, d'insecte, d'arachnide et des débris. Cette espèce à un régime omnivore à tendance ichtyophage. Par conséquent, la position de cette espèce dans la chaine alimentaire pourrait justifier la forte contamination de ses organes par les éléments métalliques. Les concentrations des métaux lourds dans les organes de poissons collectés dans cette étude sont basses comparativement aux concentrations enregistrées par Enuneku et al. (2015) dans les organes de Hemichromis fasciatus collecté dans le Delta de Niger. Ces auteurs ont noté des concentrations de cadmium de $0,80 \pm 0,13 \mathrm{mg} \cdot \mathrm{kg}^{-1}$ dans le muscle et de 1,70 \pm 0,66 mg. $\mathrm{kg}^{-1}$ dans le foie. Les concentrations moyennes de plomb des organes de poissons étudiés sont également basses aux teneurs moyennes $(1,41$ $\left.\mathrm{mg} . \mathrm{kg}^{-1}\right)$ de plomb trouvées par Youssao et al. (2011) dans les organes de Tilapia guineensis et Hemichromis fasciatus pechés dans le complexe lagunaire Nokoué au Benin. Cette différence de teneurs de métaux lourds dans les organes des poissons peut être liée au niveau de pollution. Les zones continentale et maritime à l'ouest de la lagune Ebrié semble être les moins impactées par les effluents urbains. Les concentrations

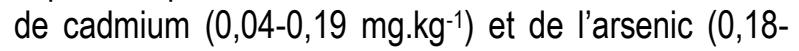
$\left.0,28 \mathrm{mg}^{\mathrm{kg}}{ }^{-1}\right)$ dans le muscle de Hemichromis fasciatus et de $T$. zillii $\times T$ Guineensis notées dans cette étude sont supérieures aux concentrations $\left(0,05 \mathrm{mg}_{\mathrm{kg}}{ }^{-1}\right.$ pour le $\mathrm{Cd}$ et $0,1 \mathrm{mg} \cdot \mathrm{kg}^{-1}$ pour l'As) recommandées par WHO/FAO $(1995,2005)$ dans les poissons comestibles. Par ailleurs, les concentrations moyennes de $\mathrm{Hg}(0,11$ $0,18 \mu \mathrm{g} / \mathrm{kg})$ et de $\mathrm{Pb}\left(0,11-0,19 \mathrm{mg}^{\mathrm{kg}}{ }^{-1}\right)$ dans le 
muscle des espèces de poissons étudiées sont inférieures à la concentration de $0,5 \mathrm{mg}_{\mathrm{kg}}{ }^{-1}$ pour $\mathrm{Hg}$ et

\section{CONCLUSION ET APPLICATION DES RESULTATS}

Les résultats de cette étude révèlent la présence des éléments métalliques dans les organes de Hemichromis fasciatus et de Tilapia. zillii x Tilapia. guineensis collectés dans les zones continentale et maritime dans la partie ouest de la lagune Ebrié. Les organes des espèces sont généralement riches en arsenic et en cadmium et pauvre en plomb et en mercure. Les valeurs élevées des métaux lourds dans les organes sont observées pendant la saison des pluies et des crues dans les zones continentale et maritime. Le foie et le rein des deux espèces sont les plus accumulateurs de métaux lourds tandis que le muscle est l'organe le moins accumulateur. Cependant, les concentrations de cadmium et de l'arsenic dans les muscles dépassent les limites $\left(0,05 \mathrm{mg} \mathrm{kg}^{-1}\right.$ pour le $\mathrm{Cd}$

\section{REMERCIEMENTS}

Les auteurs remercient le Fonds interprofessionnel de la recherche et le Conseil agricole (FIRCA) pour les

\section{REFERENCES BIBLIOGRAPHIQUES}

Adjagodo A, Agassounou DT, Ahouangnivo N, Lawani $\mathrm{R}, 2016$. Flux des polluants liés aux activités anthropiques et risque sur les ressources en eau de surface à travers le monde. Larhyss Journal, pp 7-23

Affian K, Kadio B, Djagoua EV, Digbehi ZB, Monde S., Wognin A.M., Krou D. et Mobio A., 2008. Flux de la matière en suspension du fleuve Comoé dans la zone littorale Ivoirienne. Revue CAMES, Sciences et médecine, $6: 88-93$.

Aminot $A$. et Chaussepied M, 1983. Manuel des analyses chimiques en milieu marin. CNEXO, Editions Jouve, Paris, $395 \mathrm{p}$.

Arfi R. et Guiral D, 1994. Un écosystème estuarien eutrophe : la baie de Biétri. Environnement et ressources aquatiques de Côte d'Ivoire $T$. II. Les milieux lagunaires. Ed. ORSTOM, 59-90.

Blahoua KG, Adou YE, Gogbé ZM, N'Douba V, 2017. Régime Alimentaire De Hemichromis Fasciatus (Perciformes, Cichlidae) Dans Le Lac De Barrage Hydroélectrique d'Ayamé 2 (Côte d'Ivoire). European Scientific Journal. 13(30) :126-138. Doi : 10.19044/esj. 2017. v13n30p126

Boni L, Coulibaly S, Nobah CSK, Atse B, Kouamelan EP, 2016. Physical and chemical parameters de $0,2 \mathrm{mg} \cdot \mathrm{kg}^{-1}$ pour le $\mathrm{Pb}$ recommandées par $\mathrm{WHO} /$ FAO $(1995,2005)$.

et $0,1 \mathrm{mg} \cdot \mathrm{kg}^{-1}$ pour l'As) recommandées par WHO/FAO (1995, 2005). Cette contamination des organes de poissons par les métaux lourds témoigne de l'influence des activités anthropiques, sources potentielles des éléments métalliques dans les eaux de cette partie de la lagune Ebrié. Ces fortes concentrations de cadmium et d'arsenic dans le muscle peuvent influencer la santé humaine par la consommation des poissons pêchés dans cette partie de la lagune Ebrié. Pour limiter la mortalité des poissons et garder la santé des consommateurs, il est important d'abandonner certaines pratiques culturales telles que l'utilisation abusive des produits phytosanitaires et de bateaux motorisés hors-bord dans cette partie de la lagune Ebrié.

contributions financières et au Centre de recherche océanologique (CRO) pour le suivi de ces travaux.

and nutrients in the Ebrie lagoon, (Côte d'Ivoire, West Africa): impact on fish mortality. International Journal of Research In Earth \&Environmental Sciences. 4(3) : 1-16.

Calamari D, Naeve H, 1994. Revue de la pollution dans l'environnement aquatique africain. Document technique du CPCA, N²5, Rome, FAO, p.129.

Canli M. et Atli G, 2003. The relationships between heavy metal ( $\mathrm{Cd} \mathrm{Cr} \mathrm{Cu} \mathrm{Fe} \mathrm{Pb} \mathrm{Zn)} \mathrm{levels} \mathrm{and}$ the size of six Mediterranean fish species. Environnemental Pollution. 121 : 129-136.

Claire L, Feix C, François L, 2005. Contamination des Sols. Transferts des Sols Vers les Animaux. Ed Sciences/ Ademe ; 216.

Coulibaly S, Atse BC, Koffi KM, Sylla S, Konan KJ, Kouassi NJ, 2012. Seasonal Accumulations of Some Heavy Metal in Water, Sediment and Tissues of Black Chinned Tilapia Sarotherodon melanotheron from Bietri Bay in Ebrie Lagoon, Ivory Coast. Bulletin of Environmental Contamination and Toxicology, 128: $12-522$.

Coulibaly S, Coulibaly M, Atsé BC, 2019. Contamination à l'arsenic des eaux et des sédiments des zones continentale et maritime 
de la partie ouest de la lagune Ebrié (Côte d'Ivoire). International Journal of Innovation and Applied Studies, 25(2) : 577-585.

Daniel KK, Bernard YO, Ladji M, 2016. Contamination Des Sédiments D'une Lagune Tropicale Urbaine Par Les Eléments Traces Métalliques (As, $\mathrm{Cd}, \mathrm{Cr}, \mathrm{Pb}, \mathrm{Zn}$ ) : Cas Des Baies Lagunaires De La Ville D'Abidjan (Côte D'ivoire), International Journal of Pure and Applied Bioscience. 4(6): 204-217. doi : http://dx.doi.org/10.18782/2320-7051.2428

Diomandé D, Bony KY, Edia OE, Konan FK, Gourène G, 2009. Diversité des macroinvertébrés benthiques de la rivière agnéby (Côte d'Ivoire ; Afrique de l'Ouest). European Journal of Scientific Research. pp.368-377.

Dural M, Genc E, Yemenicioglu S, Sangun MK, 2010. Accumulation of some heavy metals seasonally In: Hysterotylacium aduncum (Nematoda) and its host red sea Bream, Pagellus erythrinus(Sparidae) from Gulf of Iskenderun (North-Eastern Mediterranean). Bulletin of Environmental Contamination and Toxicology, 84: 125-131

EFSA, 2005. Opinion of the Scientific Panel on contaminants in the food chain related to the safety assessement of wild \& farmed fish. (http://www.efsa.europa.eu/EFSA/efsa_locale$1178620753812 \quad 1178620762697$.htm)

Enuneku AA, Adelusi AM, 2015. Heavy metal levels in hemichromis fasciatus from Ubeji Creek, Warri, Delta State Nigeria: implications on human health through consumption .European International Journal of Science and Technology ISSN: 2304-9693.

EPA, 2007. SW-846 test methods for evaluating solid waste. Physical and chemical methods, emission spectrometry method 6010 , OSW.

Filazi A, Baskaya R, Kum C, Hismiogullari SE, 2003. Metal concentrations in tissues of the Black Sea Fish (Mugil auratus) from Sinop-Iclimari, Turkey. Human \& Experimental Toxicology. 22: $85-87$

FOXTROT, 2012. Etude d'impact environnemental et social. Rapport final, 20-93 pp.

Gouin CA, Aka N, Adiaffi B, Bamba BS, Soro N, 2016. Pollution saisonnière des sédiments de lagune par des métaux lourds ( $\mathrm{Cu}, \mathrm{Pb}$ et $\mathrm{Zn}$ ) en zone tropicale humide : cas de la lagune Mondoukou (Sud-Est de la Côte d'Ivoire).
International Journal of Biological and Chemical Sciences, 10(2): 835-845

Inza B. et Yao K.M, 2015. Physical and chemical parameters and trace metal concentrations in the Ebrie Lagoon (Côte d'Ivoire): influence of tides and urban wastewaters. Journal of Materials and. Environmental Science. 6(5): 1321-1329.

Islam MS, Ahmed MK, Al Mamun MH, Eaton D, 2016. Health risk assessment due to heavy metal exposure from commonly consumed fish and vegetables. Environ Syst Decis DOI 10.1007/s10669-016-9592-7.

Koffi $P, 2013$. Des poissons toxiques sur le marché ? Le Nouveau Réveil $N^{\circ} 3408,1 p$.

Koffi KM, Coulibaly S, Atse BC, Kouamelan EP, 2014. Survey of Heavy Metals Concentrations in Water and Sediments of the estuary Bietri Bay, Ebrie Lagoon, Côte d'Ivoire. International Journal of Research in Earth and Environmental Sciences, 3: 1-10.

Kouassi AM, Tidou AS, Kamenan A, 2005. Caractéristiques hydrochimiques et microbiologiques des eaux de la lagune Ebrié (Côte d'Ivoire). Agronomie Africaine, 2 : 117136.

Obasohan EE, Oronsaye JAO, Obano EE, 2006. Heavy metal concentrations in Malapterurus electricus and Chrysichthys nigrodigitatus from Ogba river in Benin City. African Journal of Biotechnology, 5: 974-982.

Ploetz MD, Fitts EB, Rice MT, 2007. Differential accumulation of heavy metals in muscle and liver of a marine fish, (King Mackerel, Scomberomorus cavalla Cuvier) from the Northern Gulf of Mexico, USA. Bulletin of Environmental Contamination and Toxicology. 78:134-137.

Sama KO, Solitoke HD, Gnandi K, Afiademanyo KM, Bowessidjaou EJ, 2014. Évaluation et risques sanitaires de la bioaccumulation de métaux lourds chez des espèces halieutiques du système lagunaire togolais, URI: http://id.erudit.org/iderudit/1034695ar

Tabinda AB, Hussain M, Ahmed I, Yassar A, 2010. Accumulation of Toxic and Essential Trace Metals in Fish and Prawns from KetiBunder Thatter District, Sindh Pakistan Journal of Zoology, vol. 42(5), pp. 631-638.

Traore A, Soro G, Ahoussi KE, Bamba BS, Soro N, Biemi J, 2016. Niveau de contamination en 
métaux lourds des sédiments d'une lagune tropicale : la lagune Aghien (Sud-Est de la Côte d'Ivoire). Afrique Science: Revue Internationale des Sciences et Technologie.Vol. 10 (3), pp .73-88.

WHO/FAO, 1995. Norme générale codex pour les contaminants et les toxines présents dans les produits de consommation humaine et animale, Codex standard 193-1995, 43p.

WHO/FAO, 2005. Liste provisoire des principales espèces de poissons faisant l'objet d'un commerce international (y compris propositions concernant des concentrations maximales de plomb dans différentes espèces de poissons), Trente-septième session, La Haye, Pays-Bas, $4 p$

Wu Y, Hou X, Cheng X, Yao S, Xia W, Wang S, 2007. Combining geochemical and statistical methods to distinguish anthropogenic source of metals in lacustrine sediment : a case study in Dongjiu Lake, Taihu Lake catchment, China. Environ. Geol, 52, 1467-1474.

Yao KM, Métongo SB, Trokourey A, Yobou B, 2007. Détermination de certains paramètres de pollution dans les baies d'une lagune tropicale la lagune Ebrié Côte d'Ivoire. Journal Ivoirien d'Océanologie et de Limnologie, 4(1): 1-1

Yapi DAC, 2014.Contamination métallique, bactérienne et dommages tissulaires de quelques poissons pechés dans la lagune Aby (Côte d'lvoire). Mémoire de Thèse de l'Université Nangui Abrogoua, Abidjan, Côte d'Ivoire, 90p.

Youssao A, Soclo HH, Bonou C, Vianou K, Gbaguidi M, Dovonon L, 2011. Évaluation de la contamination de la faune ichthyenne dans le complexe lagunaire Nokoué - chenal de Cotonou par le plomb: cas des espèces Sarotherodon melanotheron, Tilapia guineensis et Hemichromisfasciatus (Bénin), Int. J. Biol. Chem. Sci. 5(2) : 595-602. 